\title{
Synchronous self-elimination of autocorrelation interference in Fourier-domain optical coherence tomography
}

\author{
Jun Ai and Lihong V. Wang \\ Optical Imaging Laboratory, Department of Biomedical Engineering, Texas A\&M University, 3120 TAMU, \\ College Station, Texas 77843-3120
}

Received June 1, 2005; revised manuscript received July 8, 2005; accepted July 13, 2005

We have developed a new algorithm and configuration for self-eliminating the autocorrelation of the object wave in Fourier-domain optical coherence tomography. A self-interferogram of the object wave is acquired synchronously with the standard interferogram of the recombined object and reference waves. The former is then subtracted from the latter after Fourier transformation. The algorithm is validated by numerical simulation and by experimental measurement of a U.S. Air Force target and a feline eye. (C) 2005 Optical Society of America

OCIS codes: $170.4500,170.4470,170.3880,170.0110,070.2590,090.2880$.

Fourier-domain optical coherence tomography (FDOCT) has drawn significant attention recently. ${ }^{1-5}$ FDOCT has been shown to offer faster A-scan speed and higher sensitivity than time-domain optical coherence tomography (TDOCT). ${ }^{6,7}$ A readout rate of $29 \mathrm{kHz}$ in a line-scan CCD camera provides an A-scan rate equivalent to hundreds of meters per second, ${ }^{3}$ which easily surpasses the grating-lensbased rapid scanning optical delay line that is widely used in high-speed TDOCT.

The main challenges to developing highperformance FDOCT are the autocorrelation (AK) interference and ghost images, both of which obscure the interpretation of images and degrade the system sensitivity. To tackle these problems, some investigators have developed full-range complex FDOCT to construct a complex field of the object wave by retrieving the phase information, which is accomplished by modulating the phase delay in the reference arm through a phase modulator or a piezo-actuator. ${ }^{3-5}$ A common problem with the phaseshifting technique, however, is polychromatic phase error, i.e., the dependence of phase shift on wavelength, which may introduce significant phase errors in ultrahigh-resolution FDOCT where the bandwidth of the source is $>100 \mathrm{~nm}$. Other potential issues include the repeatability and stability of piezoactuation, higher-order dispersion introduced by an electro-optical crystal in the phase modulator, and motion artifacts of the object. The AK selfelimination technique that we present below is tasked to eradicate all of these problems.

The standard interferogram $r o(k)$ acquired by a typical Michelson interferometer-based FDOCT can be expressed as

$$
r o(k)=\left|E_{o}(k)+E_{r}(k)\right|^{2},
$$

where $k=2 \pi / \lambda$ is the wavenumber, with $\lambda$ being the wavelength in vacuum; and $E_{o}(k)$ and $E_{r}(k)$ are the backscattered optical fields from the object and the reference mirror, respectively. Equation (1) can be expanded and rearranged as

$$
x c(k)=\operatorname{ro}(k)-\operatorname{obj}(k)-\operatorname{ref}(k),
$$

where $x c(k)=2 \operatorname{Re}\left[E_{o}(k) E_{r}(k)^{*}\right]$ is the mutual interference between the object and the reference waves, $\operatorname{obj}(k)=\left|E_{o}(k)\right|^{2}$ is the self-interferogram of the object wave, and $\operatorname{ref}(k)=\left|E_{r}(k)\right|^{2}=s(k)$ is the spectral intensity of the source. The space-domain counterpart of Eq. (2) is then obtained:

$$
X C(z)=R O(z)-\operatorname{OBJ}(z)-\operatorname{REF}(z),
$$

where $X C(z), R O(z)$, and $\operatorname{REF}(z)$ are the inverse Fourier transforms of $x c(k)$, ro $(k)$, and $\operatorname{ref}(k)$, respectively.

To help understand the AK interference, we assume that

$$
\begin{aligned}
& E_{r}(k)=E_{s}(k) \exp \left(-j k 2 z_{r}\right), \\
& E_{o}(k)=\sum_{i} a_{i}\left(z_{i}\right) E_{s}(k) \exp \left(-j k 2 z_{i}\right),
\end{aligned}
$$

and then we have

$$
\begin{aligned}
x c(k)= & s(k) \sum_{i} 2 a_{i} \cos \left(k 2 z_{i r}\right), \\
\operatorname{obj}(k)= & s(k) \sum_{i} a_{i}^{2} \\
& +s(k) \sum_{i \neq j} 2 a_{i} a_{j} \cos \left(k 2 z_{i j}\right),
\end{aligned}
$$

where $z_{r}$ and $z_{i}$ are the single-pass optical path lengths of the reference arm and the backscatters in the object arm, respectively; $z_{i r}=z_{i}-z_{r} ; z_{i j}=z_{i}-z_{j}$; $E_{s}(k)$ is the spectral amplitude of the source; and $a_{i}$ is the amplitude backscattering coefficient of the $i$ th scatterer within the object, normalized to the amplitude reflection coefficient of the reference mirror. Both $E_{s}(k)$ and $a_{i}$ are measured in the detection arm, and therefore we have already taken into account the effect of, e.g., beam splitting-recombination. The space-domain counterpart of Eqs. (5) can be obtained as 


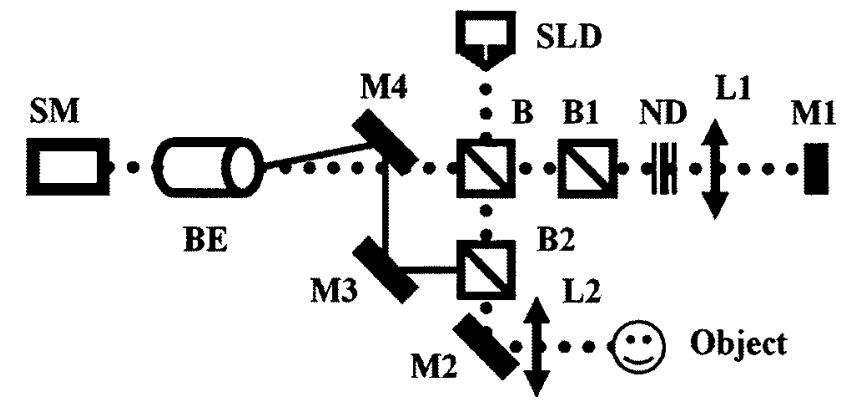

Fig. 1. Schematic of an AK self-elimination FDOCT system: SLD, superluminescent diode; B, B1, B2, beam splitters; L1, L2, lenses; BE, beam expander; ND, neutral density filter; M1-M4, mirrors; SM, spectrometer.

$$
\begin{aligned}
X C(z)= & \sum_{i} a_{i} \Gamma\left(z-z_{i r}\right)+\sum_{i} a_{i} \Gamma\left(z+z_{i r}\right), \\
\operatorname{OBJ}(z)= & \sum_{i} a_{i}^{2} \Gamma(z)+\sum_{i \neq j} a_{i} a_{j} \Gamma\left(z-z_{i j}\right) \\
& +\sum_{i \neq j} a_{i} a_{j} \Gamma\left(z+z_{i j}\right),
\end{aligned}
$$

where $\Gamma(z)$ is the AK of the source. $X C(z)$ is the crosscorrelation between the object and the reference waves, which gives the profile of the normalized amplitude reflection coefficient of the object and, therefore, is the signal that we need to extract. $\operatorname{OBJ}(z)$ is the AK of the object wave and contains not only a DC component (located at $z=0$ ) but also the AC components (located at $z \neq 0$ ) that are mixed with $X C(z)$.

Our algorithm requires that one first acquire $r o(k)$, $\operatorname{obj}(k)$, and $\operatorname{ref}(k)$ and then Fourier transform them to $R O(z), \operatorname{OBJ}(z)$, and $\operatorname{REF}(z)$. The $X C(z)$ is obtained simply by subtracting $\mathrm{OBJ}(z)$ and $\operatorname{REF}(z)$ from $R O(z)$. This is why we call the algorithm "the selfelimination of AK interference." $\operatorname{ref}(k)$ needs to be acquired only once by blocking the object arm for the entire image, whereas $r o(k)$ and $\operatorname{obj}(k)$ have to be acquired for each A-scan. The subtraction of $\operatorname{REF}(z)$ is necessary if the source is not perfectly Gaussian or if there are ripples in the spectrum ref $(k)$. Keep in mind that the Fourier transform from Eq. (2) to Eq. (3) requires uniform sampling in wavenumber. In a spectrometer, however, the spectrum is evenly spread in wavelength. Therefore, the raw spectrum must be interpolated to avoid degrading the axial resolution and SNR. ${ }^{8}$

Note that $X C(z)$ contains two parts: the object itself and the ghost (mirror) of the object. The ghost may overlap the object if the reference plane is placed improperly. By placing the reference plane outside of the object (or the region of interest), the ghost is shifted to the other side of the object and, therefore, can be removed by retaining only the positive $\mathrm{AC}$ components. The sacrifice is that the measurement range is reduced by half compared with full-range complex FDOCT.

A schematic diagram of an AK self-elimination FDOCT system is shown in Fig. 1 . The $1.6 \mathrm{~mW}$ collimated light from a superluminescent diode source at
$850 \mathrm{~nm}$ with a FWHM of $23 \mathrm{~nm}$ is split into reference and object arms by a beam splitter, B. The maximum power delivered to the object is $0.3 \mathrm{~mW}$. The B-scan is provided by a galvanometer mirror, M2 (Cambridge $6220 \mathrm{M})$. The backscattered waves from the object and reference mirror M1 are expanded by beam expander $\mathrm{BE}$ before reaching a spectrometer comprising diffraction gratings $\mathrm{G}$ (1200 line pairs/mm), an achromatic (focal length $400 \mathrm{~mm}$ ), and a CCD camera (Andor 16-bit $1024 \times 256$ array, pixel $26 \mu \mathrm{m}$ $\times 26 \mu \mathrm{m}$ ). The backscattered light of the object (the solid lines) is sampled by beam splitter B2, redirected by mirror M3, and then imaged to different row (s) in the CCD array by tilting mirror M4, which is placed just above the recombined light exiting from beam splitter B. Since $\operatorname{ro}(\lambda)$ and $\operatorname{obj}(\lambda)$ are simultaneously recorded by the CCD array (in different rows), synchronous elimination of AK is achieved. To compensate for the dispersion introduced by beam splitter B2 into the object arm, an identical beam splitter $\mathrm{B} 1$ is placed in the reference arm. The data acquisition of the CCD camera is externally triggered and synchronized with the B-scan signal by a customdeveloped LabView program. The spectrometer is able to measure a wavelength range of $47.7 \mathrm{~nm}$ with a spectral resolution of $\delta \lambda=46.6 \mathrm{pm}$ at $\lambda_{0}=850 \mathrm{~nm}$, which translates to a FDOCT measurement range of $z_{\max }=3.9 \mathrm{~mm}$ in the air according to $z_{\max }=\lambda_{0}{ }^{2} /(4 \delta \lambda)$.

To validate the capability of AK self-elimination of our FDOCT system, a U.S. Air Force 1951 positive target is imaged. The B-scan is performed across the III bar pattern and consists of 200 exposures, acquired in $4 \mathrm{~s}$. Each exposure records both $\operatorname{ro}(\lambda)$ and $\operatorname{obj}(\lambda)$ in two separate tracks, each track comprising four rows vertically binned to 1024 pixels. The raw spectra $r o(\lambda)$ is interpolated to the $k$-domain and then Fourier transformed to $R O(z)$, which is shown in Fig. 2(a), where the reference plane is at the top $(z=0)$. The first bright line down at $z=0.427 \mathrm{~mm}$ is the first surface of the target and the third line is the second surface. The optical spacing between them is $2.259 \mathrm{~mm}$, which agrees with the specified thickness $(1.5 \mathrm{~mm})$ of the target assuming a refractive index of 1.5. The second line is the AK between the two surfaces and is located at $z=2.259 \mathrm{~mm}$, which also agrees with the thickness of the target. The lateral B-scan crosses 3.5 line pairs in group 0, element 6 and covers $1.95 \mathrm{~mm}$ on the target, which agrees with the specified spatial resolution of 1.78 line pairs $/ \mathrm{mm}$. Likewise, $\operatorname{obj}(\lambda)$ is Fourier transformed to $\operatorname{OBJ}(z)$, which is then subtracted from $R O(z)$ to extract the two surfaces of the target, as shown in Fig. 2(b).

(a)
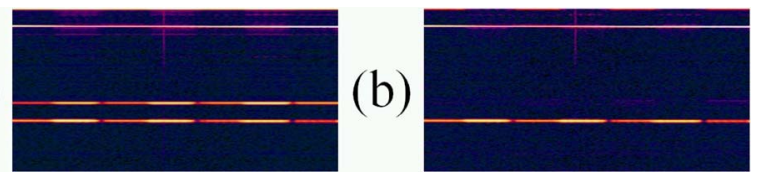

Fig. 2. (Online color) FDOCT images of a U.S. Air Force 1951 target (a) before and (b) after self-elimination of AK. The B-scan crosses the III bar pattern in group 0, element 6. Image dimensions: width (B-scan) $1.95 \mathrm{~mm}$, height (optical depth) $3.9 \mathrm{~mm}$ in air. 
Figure 3(a) is a measured ex vivo image of a feline eye. The reference plane is placed $0.18 \mathrm{~mm}$ before the front surface of the cornea. The AK of the object is clearly seen from $z=0$ to $1 \mathrm{~mm}$ in the cornea. The thick bright line at $z=2.2 \mathrm{~mm}$ is caused by ripples in the source spectrum, which were introduced intentionally to test the functionality of source AK selfelimination. Figure 3(b) is the image after AK selfelimination. It is clear that the object $\mathrm{AK}$ and the sidelobes of the source are removed completely.

The shot-noise-limited sensitivity can be estimated by $S=10 \log _{10}\left[\eta \tau P_{s} /(h \nu)\right](\mathrm{dB})$, where $h \nu$ is the photon energy, $\eta$ is the quantum efficiency, $\tau$ is the exposure time, and $P_{s}$ is the maximum optical power received by the CCD from a perfectly reflective object. ${ }^{7}$ For our FDOCT system, $\eta=\sim 0.5, \tau=11 \mathrm{~ms}$, and $P_{s}$ $=\sim 20 \mu \mathrm{W}$, and therefore the estimated value $S$ $=117 \mathrm{~dB}$. To measure the sensitivity, the reference light is first set to nearly saturate the CCD, and then neutral density filters are added in front of the object, a perfectly reflective mirror. The sensitivity is obtained by adding the A-scan signal-to-noise ratio and the round-trip attenuation of the neutral density filters in the object arm. The measured sensitivity is $112 \mathrm{~dB}$ at $0.3 \mathrm{~mm}$, and it gradually reduces to $100 \mathrm{~dB}$ at $3.7 \mathrm{~mm}$. The decrease in sensitivity with depth is caused by the finite spectral resolution of the spectrometer. $^{2}$ The measured axial resolution is $15 \mu \mathrm{m}$ in air.

We have developed a program to simulate the AK self-elimination. The simulated object is a glass slide of $1 \mathrm{~mm}$ thick. The refractive index is assumed to be 1.5. The normalized backreflection coefficient of the glass is assumed to be $a_{1}=1$ for the front surface and $a_{2}=0.96$ for the back surface. Figures $4(\mathrm{a})$ and $4(\mathrm{~b})$ show simulated $\operatorname{ro}(\lambda)$ and obj $(\lambda)$ of the glass, respectively. $\operatorname{ro}(\lambda)$ is then interpolated and Fourier transformed into $R O(z)$, which is shown in Fig. 4(c), where the reference plane is at $z=0$. The amplitude of the $\mathrm{DC}$ component is 14,758 and is truncated at 6000 to show the smaller AC peak (amplitude 4226.4) at $z$ $=1.5 \mathrm{~mm}$. The peaks at $z=1.0 \mathrm{~mm}$ and $z=2.5 \mathrm{~mm}$ correspond to the two surfaces of the glass, where the

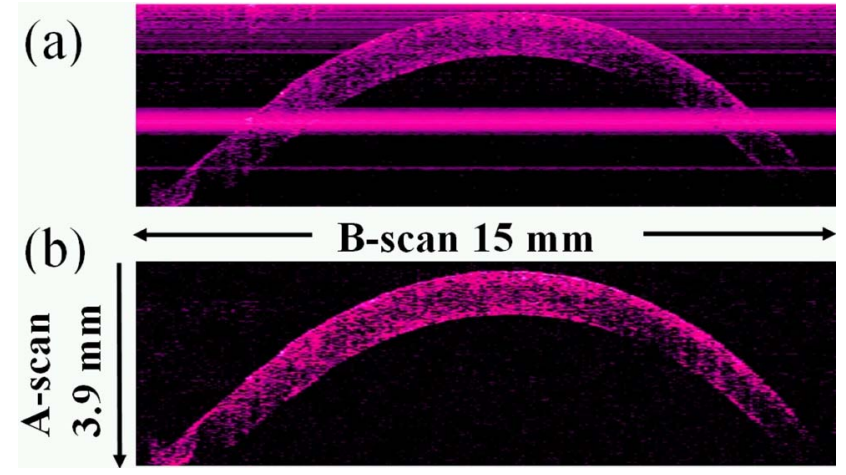

Fig. 3. (Online color) FDOCT images of a feline eye (a) before and (b) after self-elimination of AK.
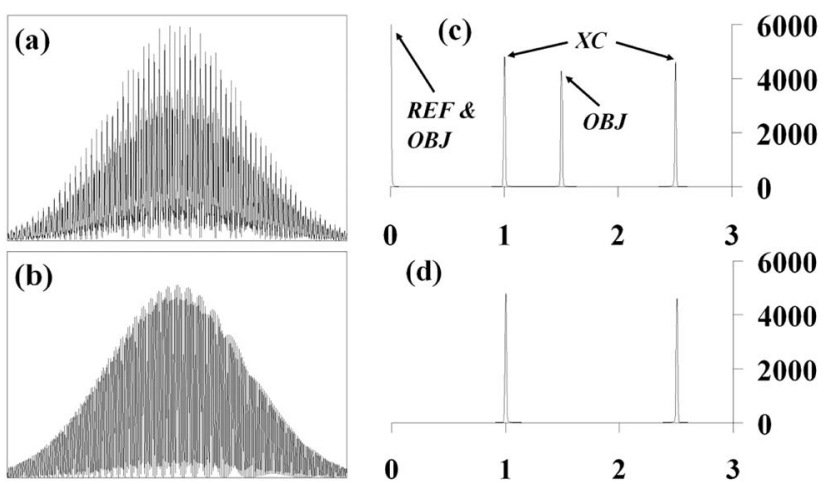

Fig. 4. Simulated (a) $\operatorname{ro}(\lambda),(\mathrm{b}) \operatorname{obj}(\lambda),(\mathrm{c}) R O(z)$, and (d) $X C(z)$ of a glass slide. The vertical scale of (b) is half that of (a). The $x$ and $y$ axes in (c) and (d) are optical depth (mm) and amplitude, respectively.

spacing between them agrees with the thickness of the glass. Likewise, $\operatorname{obj}(\lambda)$ is Fourier transformed to $\operatorname{OBJ}(z)$, which consists of two peaks: a DC with amplitude 9705.1 and an AC at $z=1.5 \mathrm{~mm}$ with amplitude 4226.3, as shown in Fig. 4(c). The location of the AC peak also agrees with the thickness of the glass. $\operatorname{REF}(z)$ is also calculated and appears as a DC peak with amplitude 5050.7, which, if added with the DC peak of $\mathrm{OBJ}(z)$, agrees well with the DC amplitude of $R O(z) . X C(z)$, shown in Fig. 4(d), is obtained by subtracting $\operatorname{OBJ}(z)$ and $\operatorname{REF}(z)$ from $R O(z)$. It is clear that the AK of both the glass and the source is completely removed.

In conclusion, we have developed a self-elimination algorithm of AK interference and built a FDOCT system capable of synchronous AK self-elimination. The algorithm is validated by numerical simulation and experimental measurement of a U.S. Air Force target and a feline eye.

We thank George Stoica for providing the feline eye specimen and John Alford for providing the CCD camera. This project is sponsored by National Institutes of Health grant R01 CA092415. L. V. Wang's email address is LWang@oilab.tamu.edu.

\section{References}

1. G. Hausler and M. W. Lindner, J. Biomed. Opt. 3, 21 (1998).

2. S. H. Yun, G. J. Tearney, B. E. Bouma, B. H. Park, and J. F. de Boer, Opt. Express 11, 3598 (2003).

3. E. Götzinger, M. Pircher, R. A. Leitgeb, and C. K. Hitzenberger, Opt. Express 13, 583 (2005).

4. J. Zhang, J. S. Nelson, and Z. Chen, Opt. Lett. 30, 147 (2005).

5. R. A. Leitgeb, C. K. Hitzenberger, A. F. Fercher, and T. Bajraszewski, Opt. Lett. 28, 2201 (2003).

6. J. F. de Boer, B. Cense, B. H. Park, M. C. Pierce, G. J. Tearney, and B. E. Bouma, Opt. Lett. 28, 2067 (2003).

7. R. Leitgeb, C. K. Hitzenberger, and A. F. Fercher, Opt. Express 11, 889 (2003).

8. C. Dorrer, N. Belabas, J.-P. Likforman, and M. Joffre, J. Opt. Soc. Am. B 17, 1795 (2000). 